\title{
Correspondence: The Alarming Need for formal Podiatry Education in Developing Countries
}

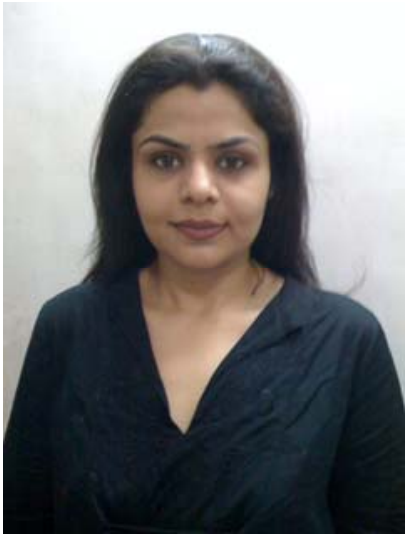

Dr. Rumneek Sodhi $1 \square$

The WHO (World Health Organization), a global health watchdog, has quoted that an estimated 180 million people worldwide have diabetes and nearly 1.1 million people succumbed to this ailment in 2005 . It comes as no surprise that majority of these ailments are prevalent in developing countries such as India, Bangladesh and Pakistan. It also estimates that the global diabetic population will grow to more than 200 million in 2010 and 330 million in 2025; with these developing countries bearing the major burnt of this epidemic in the $21^{\text {st }}$ century.

Every patient with diabetes from any origin has the potential to develop foot problems in the course of the disease; with this fact holding true at all times: A trained podiatrist is a vital member of the diabetic multidisciplinary team. The medical and nursing champions of such teams have been and would remain our greatest allies. These multi disciplinary teams are required to provide a much broader range of interventions to the whole diabetic population in a wide range of settings. The diverse work spectrum ranging from lengthy, complicated neuro-vascular assessments, nifty scalpel work, to foot surgeries are all handled by versatile, comprehensively trained podiatrists. One thing is certain-that there is definitely a place for the interested and increasingly skilled podiatrist whenever diabetes care is delivered.

It has been noted in the literature that with a rapid global increase in population growth, the aged increase in the geriatric population and ailments like diabetes and obesity, there would be an inevitable increase in the demand for the number of trained podiatrist worldwide. Physicians all over the world are striving hard to attain one common goal: The institution of the "Put Feet First: Prevent Amputations", the theme of the 2005 World Diabetes Federation. The time has arrived for a need to outline a formal training program focusing on the management of foot care and surgery for limb salvage.

A suggested training model would consisting of three different 'Tiers', however it is imperative to first identify the skills and competences that are required to perform the best clinical practice in all of these tiers. 
The first 'tier' would consist of a "fellowship program" to facilitate podiatry physician \& surgeons. They would be a part of the integrated medical system and would provide a multi disciplinary environment to improve the culture of and landscape of the health care field as a whole. They would be much better trained and more closely aligned with other medical specialties. In other words, they would be able to titrate their medicines performing their own patient histories and physicals, thus managing cases on their own. Within the USA, this model exists with the Podiatric Physicians as DPMs, but outside the USA, it could exist largely as a fellowship model in a standardized medical program.

The second tier would consist of the "podiatrist" or "podiatric clinician" serving to perform as a "physician extender". He or she is an independent practitioner and can perform minor podiatry procedures. Multi-year podiatry training in the Commonwealth countries best exemplifies this level of training. These clinicians may also provide home care to non-ambulatory foot disorder patients and serve as an "independent physician extender".

The third tier would consist of training for the "Podiatry technicians" who would be able to perform foot related assessment tests such as the foot pressure scan, biothesiometer, temp-touch etc. These technicians would serve to assist the independent clinicians in tiers 1 and 2. This training may last from weeks to months.

Podiatry, as a named field, exists in less than $10 \%$ of the nations of the world. This three-tier approach would serve as a roadmap for formal podiatry education. To achieve this goal, we would require dissemination of new ideas and inspiration from the establishment pioneers of podiatry medicine. The team approach within the profession and with other medical specialties would ensure improvement in management of foot related disorders globally and increase the number of limbs that can be salvaged in the future.

Acknowledgements: This piece is inspired under the fortunate guidance of Dr David Armstrong while I pursued my International scholar's training program at "CLEAR" institute under the aegis of Rosalind Franklin University, Chicago Illinois. The experience gained there has help me refurbish our Diabetic Wound Care Centre to provide "State of the art care" to our patients with a wide spectrum of foot ailments; with the minimal resources available in a developing country like ours where the discipline of podiatry medicine does not exist at all.

I am hoping this piece would evoke a thought process in all its readers and help spread awareness for the lack of the alarmingly \& essential need of standardized formal education in podiatric medicine and surgery throughout the world.

${ }^{1}$ Dr. Rumneek Sodhi , Consultant in Charge, Diabetic Foot Care Centre, Department of Vascular \& Endovascular Surgery, Sir Ganga Ram Hospital, New Delhi, India. Email: rumneeksodhi@gmail.com 